\title{
SÍNTESIS BIBLIOGRÁFICA DE LA VEGETACIÓN Y CLIMA DE LA PENÍNSULA IBÉRICA DURANTE EL NEÓGENO
}

\author{
Eduardo BARRÓN ${ }^{1}$, M. Rosario RIVAS CARBALLO ${ }^{2}$ y María F. VALLE ${ }^{2}$ \\ ' Dpto. y UEI de Paleontología. Facultad de CC Geológicas e Instituto de Geología Económica. UCM-CSIC, 28040, Madrid. \\ ${ }^{2}$ Dpto. de Geología (Paleontología). Facultad de Ciencias. Universidad de Salamanca. c/ del Parque s/n, 37008 Salamanca.
}

\begin{abstract}
Barrón, E., Rivas Carballo, M.R. y Valle, M.F. 1996. Síntesis bibliográfica de la vegetación y clima de la Península Ibérica durante el Neógeno. [Bibliographic synthesis of the vegetation and clime of the Iberian Peninsula during the Neogene]. Revista Española de Paleontología, N Extraordinario, 225-236. ISSN 0213-6937.
\end{abstract}

\begin{abstract}
In this paper all of the studies about the neogene floras of the Iberian Peninsula, carried out up to now, are synthesized. Conclusions about the vegetational and climatic evolution during the Neogene are not drawn.
\end{abstract}

Keywords: Synthesis. Bibliography. Paleobotany. Palynology. Neogene. Spain. Portugal.

\section{RESUMEN}

\begin{abstract}
En el presente trabajo se realiza una síntesis bibliográfica de todas las publicaciones, llevadas a cabo hasta el momento, sobre la flora del Neógeno en la Península Ibérica, exponiéndose las conclusiones sobre la evolución de la vegetación y el clima durante este período de tiempo. Se ha respetado siempre la terminología, tanto cronológica como taxonómica utilizada por los autores consultados.
\end{abstract}

Palabras clave: Síntesis bibliográfica. Paleobotánica. Palinología. Neógeno. España. Portugal.

\section{INTRODUCCION}

El estudio de la Paleobotánica en España comenzó a desarrollarse, de forma tímida, en la segunda mitad del siglo pasado, primeramente sobre materiales carboníferos, a causa de las prospecciones mineras para la obtención de carbón. Su estudio se llevo a cabo fundamentalmente por investigadores extranjeros. El descubrimiento y descripción de floras terciarias fue posterior.

El primer trabajo que cita vegetales terciarios españoles es el realizado por Areitio y Larrinaga en 1874. Este autor enumera dos especies de gimnospermas y diez de angiospermas procedentes de los yacimientos de Lorca, Hellín, Baños de Mula, Zarra y montes de Totana. Los ejemplares citados en este trabajo fueron cedidos por los coleccionistas: condesa de Oñate, Cánovas y Bosca. Curiosamente, todavía no se han estudiado estos afloramientos desde un punto de vista de los macrorrestos vegetales.

$\mathrm{Al}$ contrario de lo que ocurre con la macroflora, los estudios palinológicos referidos al Neógeno peninsular son recientes. Los primeros trabajos sobre el tema son los de Diniz (1965), acerca del Plioceno de Rio Maior (Portugal), y Medus y Nonn (1963), que analizan el limite Tortoniense-Pontiense en Galicia. A partir de estas fechas, se han publicado un elevado numero de trabajos que contemplan tanto el ámbito marino como el continental, centrándose la mayoría de ellos en el Mioceno Superior y Plioceno.

El objetivo de este trabajo es poner de manifiesto los estudios que hasta el momento se han realizado acerca de la vegetación neógena de la Península Ibérica, respetando en todo momento citas originales, tanto lo referente a las denominaciones taxonómicas efectuadas en su momento por los autores, como la cronología (Fig. 1).

Las dataciones realizadas por los autores revisados se van a respetar en el texto, ya que las plantas neógenas no son buenos marcadores cronoestratigráficos, y usualmente, los yacimientos en donde se han colectado los restos vegetales han sido datados a partir de otros fósiles tanto marinos como continentales. Razón por la cual, en este trabajo se utilizan los pisos de ambas escalas.

\section{MACROFLORA}

Las floras neógenas de la Península Ibérica se localizan, quizás por falta de prospecciones como ya hemos comentado anteriormente, en el área mediterránea y en Portugal (Figs. 2 y 3). Se deben considerar como una excepción la flora de Épila (Zaragoza) datada por Ferrer et al. (1993) como del Aragoniense medio/superior y la de Frías (Burgos) considerada Mioceno Superior por Álvarez Ramis y Fernández Marrón (1975).

\section{Mioceno Inferior}

A finales del Oligoceno en Europa Occidental predominó una flora de tipo subtropical cálida, con taxa adaptados a la existencia de períodos de sequía. 


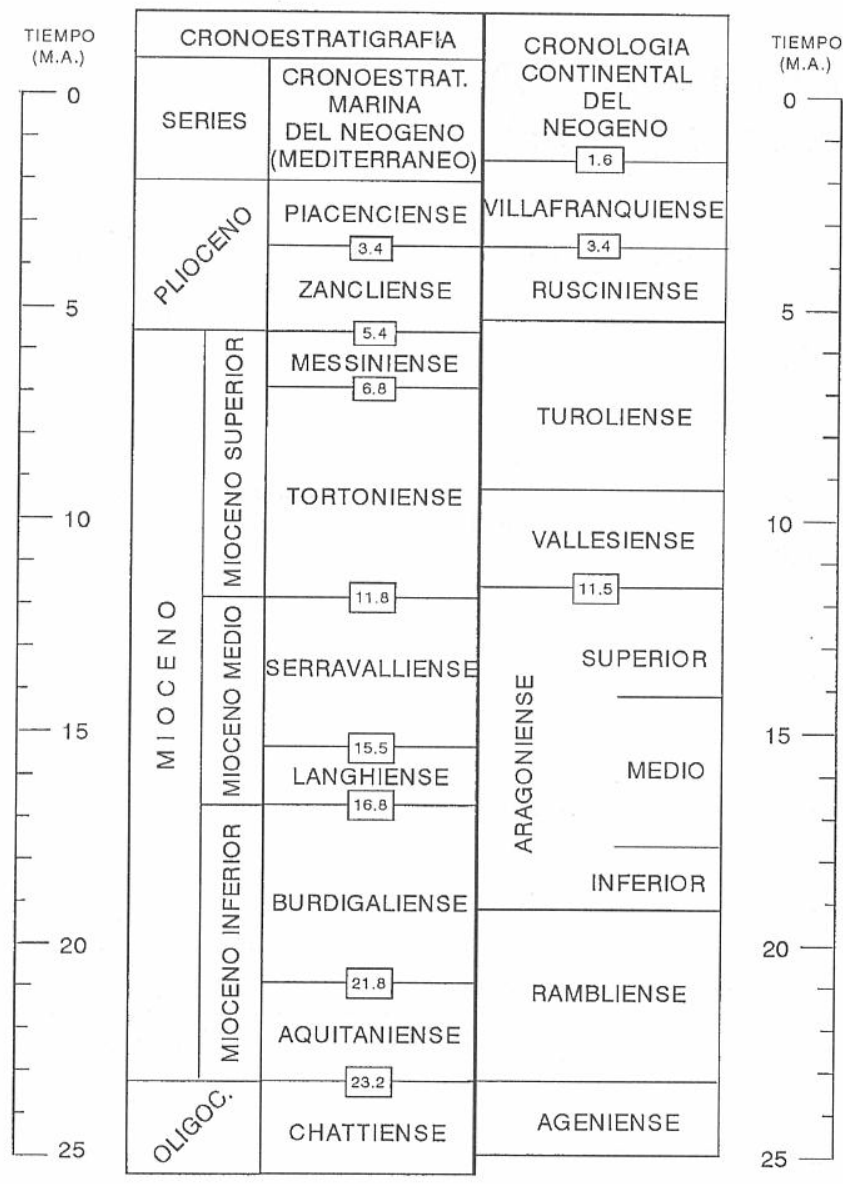

Figura 1. Tabla cronoestratigráfica del Neógeno según Calvo et al. (1993).

En la provincia de Castellón destaca el yacimiento de Ribesalbes, datado como Aquitaniense por Fernández Marrón (1971). Este afloramiento debería ser asignado al Rambliense, ya que los materiales que lo rellenan son de tipo continental al hallarse ubicado en una antigua cuenca lacustre. En sus sedimentos se han hallado un alto porcentaje de plantas ligadas a zona húmedas (Taxodium, Populus, Typha, Potamogeton), y de una forma más escasa, taxa con adaptaciones a la xericidad, que habitaban zonas secas (Pinus, Acacia, Caesalpinia) (Fernández Marrón y Álvarez Ramis, 1967; Fernández Marrón, 1971, 1979; Vigón, 1980).

En el Burdigaliense inferior se desarrolló en las proximidades de Martorell (Barcelona) una flora donde existieron vegetales adaptados a soportar estaciones secas prolongadas, debiéndose destacar los géneros Mimosa, Cassia, Robinia y Colutea, todos ellos de la familia Fabaceae (Sanz de Siria Catalán, 1981b). Según este autor la flora de Martorell presenta cierto parecido con las del Oligoceno de la cuenca de Cervera, y sobre todo con los del yacimiento de Sarreal, apreciándose en ella un aumento importante en el numero de restos y especies de Fabaceae, y una clara disminución de Lauraceae. Estos cambios hacen pensar en un aumento de aridez.

Los yacimientos de Rubielos de Mora (Teruel), atribuidos al Orleaniense (zona MN4) (De Bruijn et al., 1992), a diferencia del de las proximidades de Martorell, han proporcionado taxa fundamentalmente mesófilos. La vegetación correspondería a un bosque mixto formado por Aceraceae, Myricaceae, Betulaceae, Ulmaceae, Juglandaceae y Pinaceae (Álvarez Ramis y Fernández Marrón, 1994).

En Portugal se han colectado macrorrestos vegetales en la región de Lisboa-Almada en afloramientos correspondientes al Aquitaniense y Burdigaliense (Pais, 1979):

- En el Aquitaniense de la región vestibular de la cuenca del Tajo, se han colectado algunos restos vegetales asignables a los géneros Magnolia, Cornus y Salix (Pais, 1979). Aunque el primer género indica condiciones ambientales cálidas, no se han encontrado, como en el caso de Ribesalbes, ni coníferas ni leguminosas que indiquen condiciones de sequía.

- Se deben mencionar dos localidades atribuidas al Burdigaliense:

a) Areeiro, en donde Pais (1979) señala la presencia de leguminosas, Pinus y árboles de ribera.

b) Quinta do Bacalhau, aquí se han colectado taxa de tipo tropical o subtropical (Heer, 1881; Pais, 1979).

Finalmente, debemos mencionar el afloramiento de Ponte del Sor, datado como Burdigaliense por la presencia de Acerates longipes Heer (Pais, 1973b). En esta localidad también se han hallado maderas (Pais, 1972, 1973a).

La presencia en los yacimientos portugueses de Pinus y leguminosas, como Podogonium, parecen sugerir períodos de sequía, aunque quizás éstos no fueran tan acentuados como los que debieron existir durante la deposición de los sedimentos de Martorell, ya que se han hallado un buen numero de taxa mesófilos.

Últimamente se han hallado en Épila (Zaragoza), en un afloramiento del Aragoniense medio/superior, un escaso conjunto de restos vegetales (una especie de helecho y fragmentos de hojas de palmeras y monocotiledoneas) (Ferrer et al., 1993). El hallazgo de estos ejemplares parece indicar un clima tropical, todo lo mas subtropical, sin poder precisar la existencia o no de una estación seca.

\section{Mioceno Medio}

Desde un punto de vista paleobotánico, el Mioceno Medio se encuentra escasamente representado. Son reseñables los afloramientos de la depresión del Vallès-Penedès (Barcelona).

La flora encontrada se destaca por la gran abundancia en especies de Fabaceae incluidas en los géneros Colutea, Acacia, Caesalpinia, Cassia, Podogonium, Psoralea, Tephrosia, Gledistchia y Mimosa. También hay que destacar la presencia de restos asignables a los géneros Bumelia, Sapindus, Diospyros y Sapotacites. La presencia de estos taxa indica que la flora que se desarrolló durante el Mioceno Medio en esta zona de Cataluña, debió ser de tipo tropical o subtropical seco. Los restos foliares que se han colectado tienen un marcado carácter xérico.

Los afloramientos estudiados hasta el momento son:

1. Cercanías de Martorell, Langhiense-Serravaliense (Sanz de Siria Catalán, 1982a).

2. Rubí, Langhiense-Serravaliense, el cual presenta una composición florística similar al de la localidad anterior, aunque hay que destacar la abundancia de restos foliares atribuibles al género Celastrus, también indicador de climas secos y calurosos (Sanz de Siria Catalán, 1988). 


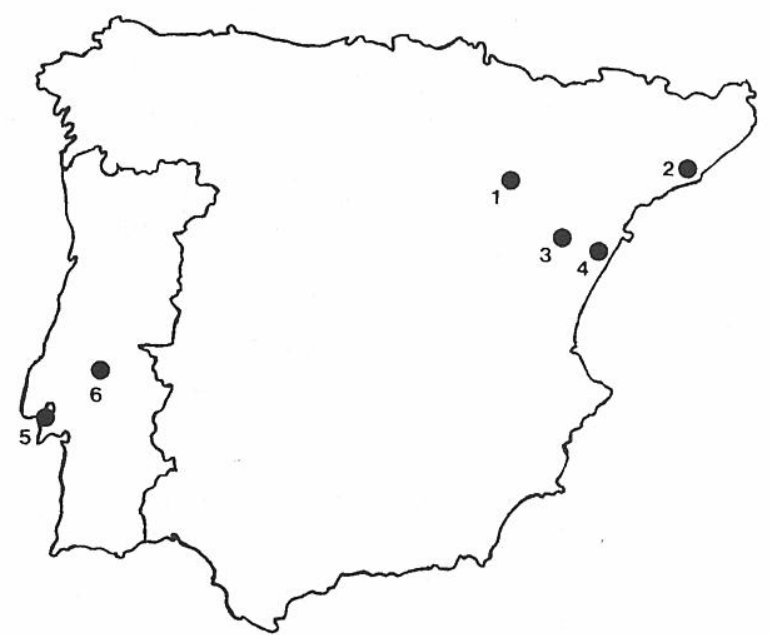

A

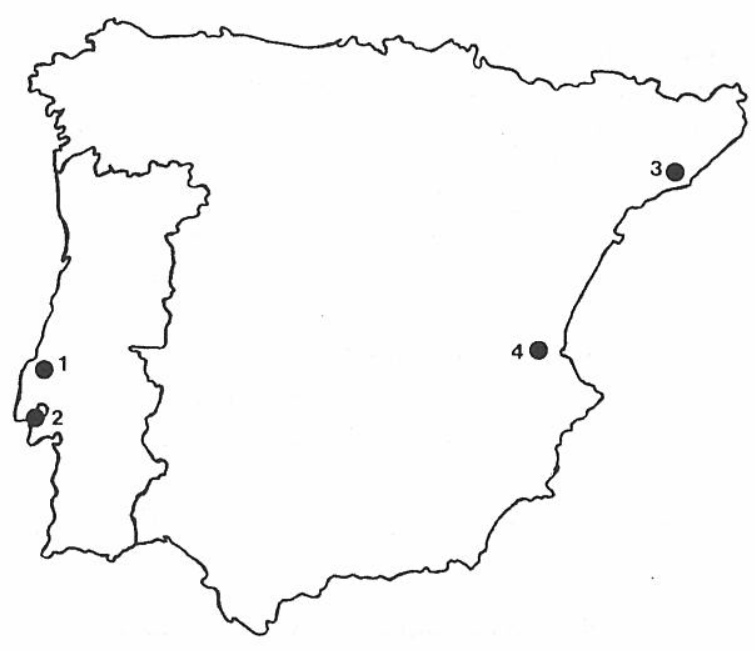

B

Figura 2. Localización geográfica de los yacimientos de macroflora. A: Mioceno Inferior: 1. Épila; 2. proximidades de Martorell; 3. Rubielos de Mora; 4. Ribesalbes; 5. región de Lisboa-Almada (Valle del Tajo, Areeiro y Quinta do Bacalhau); 6. Ponte del Sor. B: Mioceno Medio: 1. Leiria; 2. región de Lisboa-Almada (Valle de Chelas, Alaresmada); 3. Vallès-Penedès; 4 . Buñol.

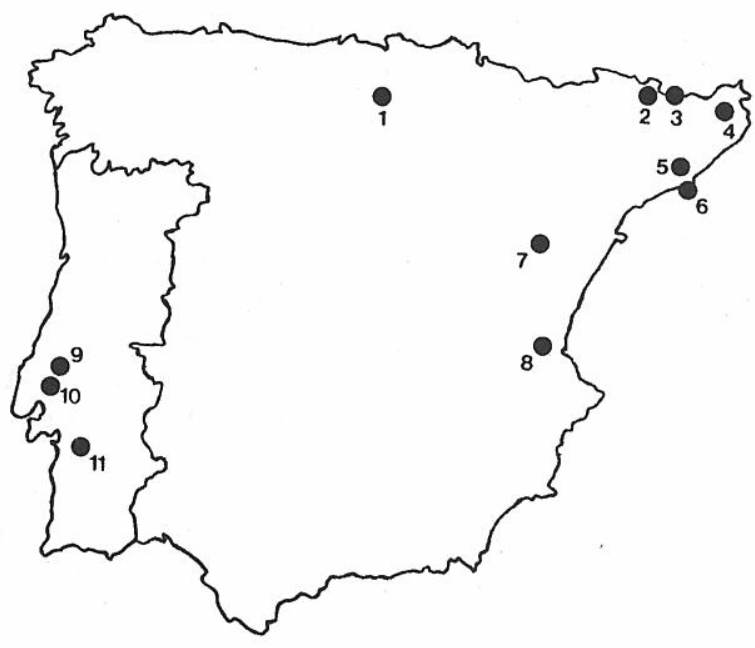

A

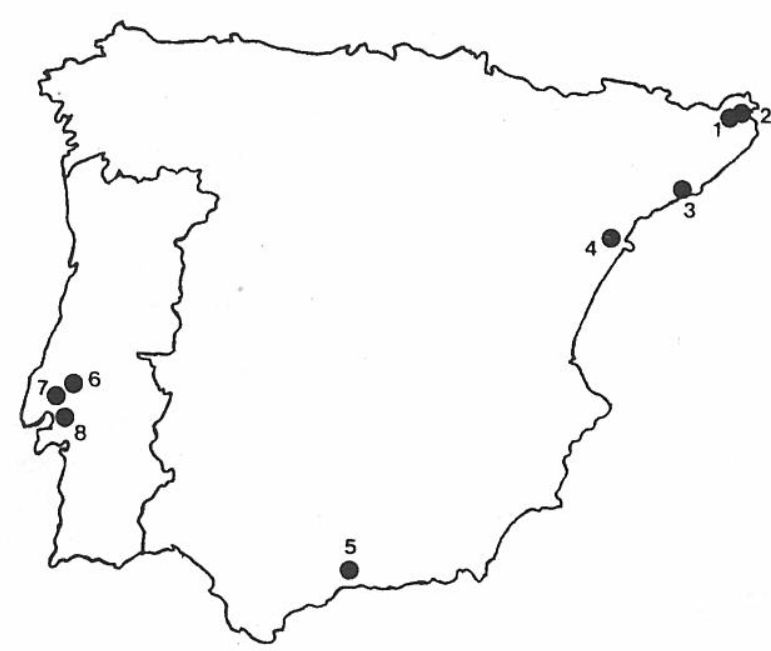

B

Figura 3. Localización geográfica de los yacimientos de macroflora. A: Mioceno Superior: 1. Frías; 2. La Seo de Urgel; 3. La Cerdaña; 4. La Bisbal; 5. Vallès; 6. Montjuïc; 7. Libros; 8. Olocau; 9. Valles de Santarém y Carros; 10. Azambuja; 11. Esbarrondadoiro y Valle de Coelheiros. B: Plioceno: 1. Crespià; 2. Ciurana; 3. Baix Llobregat; 4. Tortosa; 5. Los Tejares; 6. Valle de Santarém; 7. Río Maior; 8. Montijo. 
3. Alrededores de Sant Sadurni D'Anoia, SerravalienseTortoniense (Sanz de Siria Catalán, 1979, 1983-1984b). En este afloramiento dominan numéricamente las Fabaceae anteriormente mencionadas.

En el Langhiense del Valle de Chelas (Portugal), Pais (1979) señala la presencia de fragmentos de madera mal conservados, asignables a varios géneros de dicotiledoneas. La presencia de Pinus, Tamaricoxylon y Podogonium que indican, como en el caso de los afloramientos catalanes, un clima cálido y seco. En esta misma época, Teixeira (1973b) reseña la existencia de Pinus a partir de sus conos en Leiria y Almada.

Álvarez Ramis et Doubinger (1981) describen unos niveles con restos vegetales en Buñol (Valencia), que, tras su estudio, sitúan entre la parte alta del Mioceno Medio y el Mioceno Superior. Esta datación no está de acuerdo con los datos faunísticos que asignan estos afloramientos al Mioceno Inferior (zona MN4) (De Bruijn et al., 1992). Los taxa citados por Álvarez Ramis et Doubinguer (op. cit.) revelan un clima seco, quizás no tanto como el de la cuenca del Vallès-Penedès, donde son reseñables, además de las ya citadas Fabaceae y Celastraceae, especies mesófilas de los géneros: Betula, Alnus, Fagus, Populus y Salix. Sin embargo, la presencia del genero Pinus, representado por cinco especies continua indicando la existencia de períodos de sequía. Las autoras anteriormente citadas comparan la flora de Buñol con la del Sarmatiense de Oehningen (Suiza).

\section{Mioceno Superior}

Durante el Serravaliense el Paratetis se aisla de la zona mediterránea occidental gracias a la formación de un brazo de tierra en una zona que abarca parte de Asia Menor, por lo que el Mar Mediterráneo comienza, en el Tortoniense, a presentar una configuración parecida a la actual. Como consecuencia de esto, se produjo una variación en las condiciones ambientales que reinaban en la Península.

El yacimiento de Can Llobateres, asignado al Vallesiense inferior (zona MN9) (De Bruijn et al., 1992), se encuentra en la Cuenca del Vallès-Penedès. El registro paleontológico obtenido en este afloramiento parece señalar que la zona estuvo poblada en esta época por bosques que se desarrollaban cerca de zonas húmedas. Hay que destacar la abundancia de siconos asignables al genero Ficus recogidos en la zona (Álvarez Ramis, 1975).

Según Sanz de Siria Catalán (1985), el yacimiento de macrorrestos vegetales más importante del Vallès es el de Mas Rampinyo, de materiales de edad Tortoniense (Almera, 1891), donde se han hallado unos pocos restos foliares relacionables con varios géneros de la familias Fabaceae, Lauraceae, Myricaceae y Sapindaceae (Sanz de Siria Catalán, 1985). Según este ultimo autor, parece que las condiciones ambientales bajo las cuales se desarrollaron estas plantas, fueron bastante benignas y no muy húmedas.

Los afloramientos de la móntaña de Montjuïc han sido estudiados por varios autores (Almera, 1899; Vicente i Castells, 1988), quienes los dataron como correspondientes al Tortoniense. Según Sanz de Siria Catalán (1985), éstos pueden correlacionarse con el yacimiento de Can Llobateres, que como hemos apuntado anteriormente fue asignado al Vallesiense inferior.
En el Tortoniense de Quinta da Barroca (Portugal) fueron halladas un conjunto de piñas, con afinidades a las de los pinos actuales de California, que se describieron como Pinus o'donnelli (Teixeira, 1947c). Parece ser que en Portugal durante este período la influencia marina se atenúa, haciéndose mas secas las condiciones climáticas, por lo que los pinares pudieron tener cierta relevancia en los ecosistemas de la zona.

En Esbarrondadoiro, (Odivelas, Portugal), se encontró un nivel tortoniense con flora (Teixeira, 1952b). Sin embargo, los taxa hallados son muy escasos para llegar a ninguna conclusión de tipo climático.

Los yacimientos situados en los depósitos continentales del Valle del Tajo (Portugal), han proporcionado un buen numero de restos vegetales. No obstante, es difícil establecer el orden cronoestratigráfico de éstos, aunque su composición en flora no es muy diferente (Pais, 1979). Los afloramientos del Valle de Santarém y Valle de Carros, presentan una flora con muy pocos taxa (Teixeira, 1947b, 1952a), que recuerda a la de Esbarrondadoiro. Concretamente, los yacimientos del Valle de Santarém se han atribuido, a partir del estudio de micromamíferos, al Astaraciense (zonas MN7+8) (De Bruijn et al., 1992).

En el Vallesiense inferior de Portugal son reseñables las floras de Archino y Azambuja (Heer, 1881; Teixeira, 1946) en donde destacan, como en los yacimientos portugueses anteriormente citados, fanerófitos ligados a medios húmedos como Salix y Populus, y plantas acuáticas.

Representante de la parte media del Vallesiense es la flora de La Bisbal (Baix Empordá, Gerona) (Sanz de Siria Catalán, 1981a). En este yacimiento abundan los restos foliares de árboles, lo que quizás indique que la región estuvo cubierta en el pasado por bosques más o menos densos. Sanz de Siria Catalán (1985) señala que en la Bisbal abundan los taxa perennifolios (25\% de las impresiones recogidas), aunque el numero de éstos es inferior al de los caducifolios. Es destacable la presencia del genero Paliurus y de algunas especies de Quercus con hojas coriáceas, los cuales apuntan la existencia de períodos de sequía. La colecta de distintas especies de Lauraceae y restos foliares de árboles como Platanus, ponen de manifiesto la escasa incidencia de heladas en los meses mas fríos.

Floras peninsulares correspondientes al Vallesiense son las de las cuencas de la Seo de Urgel y la Cerdaña. No obstante, hay que señalar que estas se desarrollaron en zonas montañosas, pues los Pirineos se habían elevado tiempo atrás. El que las asociaciones vegetales de estas dos cuencas se desarrollaran en zonas de montaña nos hace comprender el elevado numero de taxa caducifolios encontrados, ya que los vegetales que allí habitaron estuvieron adaptados a condiciones mas frías producidas por la altitud. Por esta razón, se explica la no presencia de taxa como las Arecaceae que sí abundaban en regiones mas bajas en el mismo período, como indica el registro palinológico.

La mayoría de los yacimientos de vegetales de la cuenca de la Seo de Urgel están estratigráficamente muy próximos al de Ballestar, datado a partir de restos de mamíferos como Vallesiense superior, por lo que Sanz de Siria Catalán (1980b) piensa que deben pertenecer a este período. La flora del Vallesiense de la Seo de Urgel esta principalmente compuesta por especies caducifolias. No obstante, la presencia de 
especies de Fabaceae, Simaroubaceae, Proteaceae, Moraceae, Myrsiniaceae y Sapindaceae, nos indica unas temperaturas muy benignas, y quizás períodos de sequía.

La flora de la Cerdaña, datada por Álvarez Ramis y Golpe-Posse (1981) como Vallesiense-Turoliense, está compuesta principalmente por árboles caducifolios propios de climas templados (Rérolle, 1884-1885; Villalta y Crusafont, 1945; Menéndez Amor, 1955; Sanz de Siria Catalán, 1980a, Álvarez Ramis y Golpe-Posse, 1981; Barrón, 1992a, 1992b; Barrón and Diéguez, 1994). En algunos de sus afloramientos clásicos abundan relativamente los restos foliares de Lauraceae. Esto puede ser debido tanto a un problema tafonómico de acumulación de un tipo de resto en una zona determinada de la cuenca, como a la sucesión de períodos cálidos y fríos que permitieran la emigración de distintos taxa.

Según Álvarez Ramis (1981), el análisis cuantitativo de los taxa encontrados en la Cerdaña, junto con el de morfologías foliares (superficie de restos foliares y tipo de margen de éstos), indica que en la zona hubo un clima montano subtropical seco. Nosotros tras el análisis de la flora que hemos plasmado en distintos trabajos (Barrón, 1992a, 1992b; Barrón and Diéguez, 1994) pensamos que las condiciones climáticas eran templado-cálidas con un índice de precipitaciones elevado.

En la bibliografía hay citados un conjunto de floras insuficientemente conocidas asignadas al Mioceno Superior, estas son:

- Olocau (Valencia), en donde aparecen restos foliares de árboles templados de tipo caducifolio. Fue considerado por Fernández Marrón (1970), como Pontiense.

- Ullastrell, Viladecaballs, cercanías de Terrasa y Capilla de Santa Margarida (Barcelona), atribuidos al Mioceno Superior (Sanz de Siria Catalán, 1985). En estos afloramientos se han colectado restos indeterminados de vegetales y Typha.

- Turo de Montgat (Barcelona), donde se han encontrado únicamente cuatro taxa vegetales que no son suficientes para proceder a su datación, aunque Sanz de Siria Catalán (1985) lo considera Pontiense.

- Frías (Burgos), estudiada por Álvarez Ramis y Fernández Marrón en 1975. Estas autoras describen un conjunto de restos foliares fragmentarios y datan el yacimiento como Mioceno Superior.

- Libros (Teruel), aquí se han descrito contados ejemplares de restos vegetales (Meléndez Meléndez, 1945; Fernández Marrón, 1972). El último autor expresa que la cuenca esta datada como Mioceno Superior.

- Valle de Coelheiros (Portugal), se han localizado escasos ejemplares correspondientes a cuatro especies de dicotiledóneas arbóreas (Pais, 1983).

\section{Plioceno}

Por el momento no se han encontrado floras en sedimentos del Plioceno Inferior ibérico. Sin embargo, en Portugal las floras pliocenas de Montijo y el Valle de Santarém (Teixeira, 1947a, 1952c) no tienen una datación precisa. La composición de la flora del Valle de Santarém recuerda a la del Mioceno Superior de la Cerdaña, aunque presenta elementos termófilos de tipo mediterráneo como Nerium.

Se han estudiado pocas flora del Plioceno Superior, tres de ellas se localizan en Cataluña y han sido estudiadas pri- meramente por Almera (1891, 1894), y posteriormente por: Álvarez Ramis (1977), Álvarez Ramis et Fernández Marrón (1984) y Sanz de Siria Catalán (1982b, 1983-1984a, 1987).

Los yacimientos de macroflora en Cataluña se distribuyen por el Baix Llobregat (Barcelona), Ciurana (Gerona) y por los alrededores de Tortosa (Tarragona). Todos ellos tienen en común la predominancia de taxa arbóreos, la mayoría de ellos detectados ya en el Vallesiense-Turoliense de la Cerdaña, que se pueden dividir en tres grupos:

- árboles caducifolios de tipo templado como Acer, Alnus, Castanea, Fagus, Juglans, Platanus, Populus, etc...

- árboles de hoja lauroide: varias especies de Lauraceae pertenecientes a los géneros Laurus, Persea, Cinnamomum, Benzoin, y quercíneas como Quercus drymeja Ung.

- árboles y arbustos de tipo mediterráneo: Chamaerops humilis L., Quercus ilex L., Quercus coccifera L., Buxus sempervirens L., etc...

En el yacimiento de Papiol abundan con un $47 \%$ los taxa de hojas lauroides (Sanz de Siria Catalán, 1983-1984a, 1987), lo cual indica un clima de tipo subtropical con gran cantidad de humedad ambiental. Es reseñable la presencia de taxa de tipo mediterráneo, lo que señala la existencia de períodos de sequía. Estos taxa mas tarde fueron incrementando su numero lo que indica un cambio en las condiciones climáticas durante el último período del Plioceno y Cuaternario, conformando lo que es la flora actual de la región. Las floras pliocenas catalanas están datadas como Plaisancienses.

En este mismo período, Menéndez Amor (1951) cita en Los Tejares (Málaga) la existencia de una piña que determina como Pinus (pinaster) pseudo-pinea SAP., especie relacionada con el $P$. pinea $L$. mediterráneo, pero no da más referencias sobre otros restos vegetales. Hasta el momento, no hemos encontrado más citas de floras pliocenas en Andalucía.

A finales del Plioceno, las condiciones climaticas se recrudecen. Por esta razón, desaparecen casi todas las Lauraceae (destaquemos la presencia reseñada por Teixeira, 1973a, de algunos representantes de esta familia en Rio Maior, Portugal), y taxa termófilos como Liquidambar, Ginkgo, Platanus, etc, incrementándose el numero de taxa mesófilos, y entre estos, los que en la actualidad habitan en el entorno mediterráneo. En la Península Ibérica debemos destacar la flora de Crespià (Gerona) (Villalta y Vicente, 1972; Roiron, 1983). En esta localidad se han colectado un conjunto de taxa como Quercus cerris L. y Carpinus suborientalis Sap., especies cuyos descendientes hoy en día integran comunidades vegetales del Mediterráneo oriental.

\section{MICROFLORA}

Realizar una síntesis sobre los análisis palinológicos puede resultar complejo, en primer lugar por la gran cantidad de taxa que se manejan, incluso en una sola muestra. Por otra parte, no suelen referirse a localidades concretas sino a cuencas (grandes superficies) o sondeos (grandes profundidades) que ofrecen una mayor amplitud cronoestratigráfica. Estos últimos no siempre son llevados a cabo por los mismos autores, lo que dificulta la condensación de las conclusiones. De 


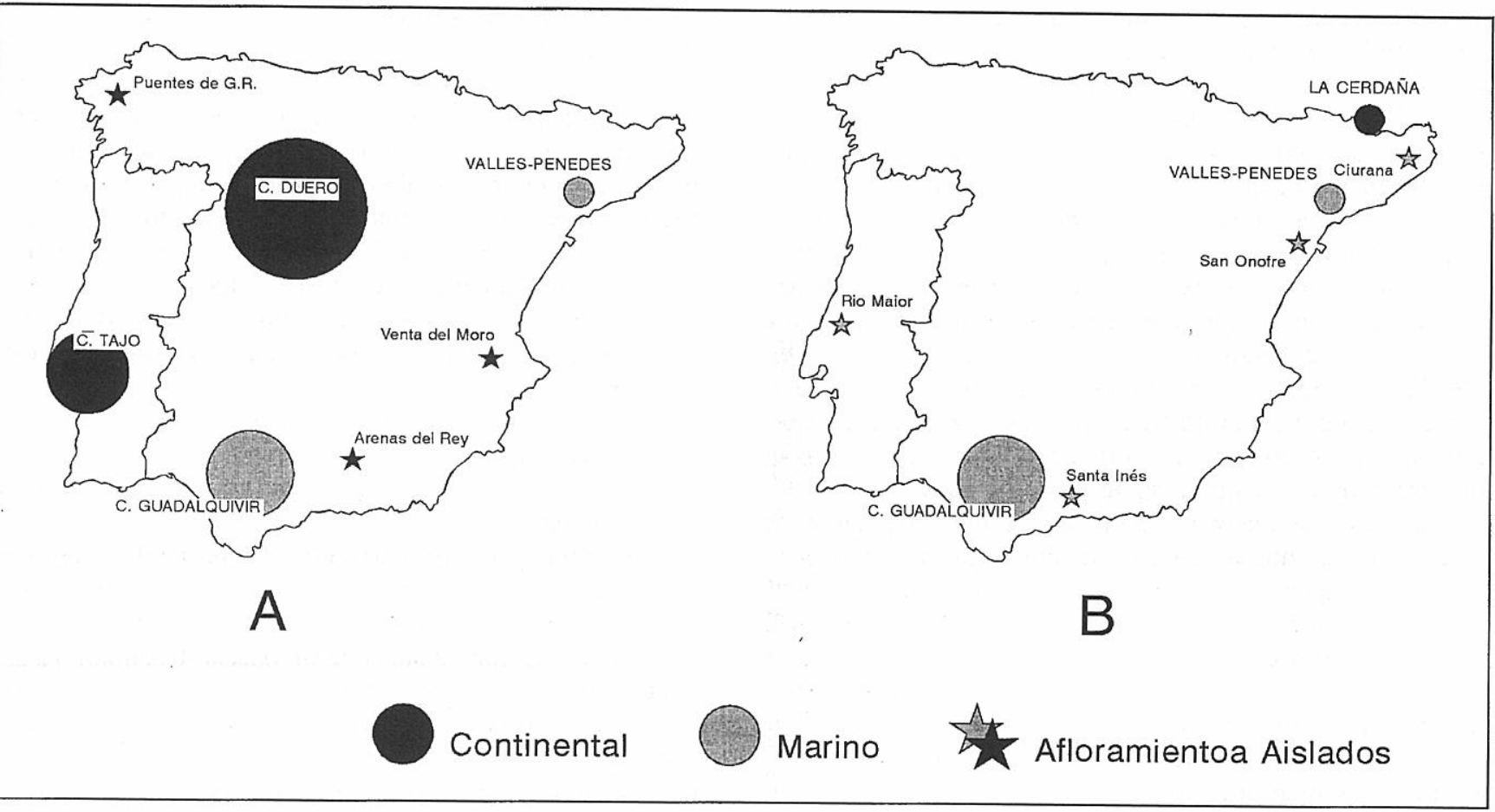

Figura 4. Localización geográfica de los yacimientos neógenos de microflora: A-Mioceno; B-Plioceno.

este modo, la síntesis de los microrrestos se ha realizado por zonas geográficas (Fig. 4), haciendo hincapié en las referencias bibliográficas referidas a cada una de ellas.

\section{DEPRESIÓN DEL DUERO}

Se trata de una cuenca muy extensa, que ha sido estudiada a lo largo de varios años. Sin embargo, los resultados obtenidos en casi todos los afloramientos coinciden entre sí, tanto en lo que se refiere a la vegetación como al clima: del estudio palinológico se desprende el paso de un paisaje de estepa muy pobre dominado por Compuestas y Amaranthaceae-Chenopodiaceae (Aragoniense superior), a otro de aspecto similar a la estepa arbolada (Vallesiense) en la que dominarían por igual árboles y herbáceas. Entre el componente arbóreo habría que distinguir una formación planifolia de ribera, constituída por fresnos y alisos, y otra con una dependecia hídrica mucho menor, dominada por Quercus. Se puede decir que las formaciones arbóreas eran ya de tipo mediterráneo.

La formación herbácea presente no sería ya una estepa pobre sino una estepa típica de gramíneas que se distribuye entre los conjuntos arbolados creando un mosaico; sin embargo, en esta unidad la estepa dejaría paso a amplias praderas de carácter húmedo que predominarían sobre ella en el paisaje. En algunas zonas de la depresión se han identificado extensos sistemas lacustres que sostendrían una variada vegetación acuática y ribereña, cuyos límites dependerían de la estacionalidad del clima.

Los afloramientos estudiados hasta el momento son:

- Torremormojón (Palencia): (Rivas Carballo, 1985, 1987; Rivas Carballo y Valle, 1986), es una de las más significativas de la cuenca; según los datos paleontológicos aportados por García Moreno (1983), López Martínez y Sanchiz (1982) y López Martínez et al. (1985), la serie corresponde al Aragoniense superior/Vallesiense. Los niveles basales de este afloramiento se apartan del esquema general de la cuenca, puesto que en ellos aparecen numerosos elementos megatérmicos (Nyssa, Taxodium, Clethraceae-Cyrillaceae...) propios de zonas pantanosas subtropicales.

- Sector Sur-Oriental: (Rivas Carballo, 1989, 1991a, 1991b). Este sector comprende numerosos afloramientos incluidos en el área Peñafiel-Aranda de Duero, que se distribuye por las provincias de Burgos, Valladolid, Segovia y Soria, y corresponden al Aragoniense superior y Vallesiense (Armenteros, 1985).

- Peñafiel (Valladolid): (Rivas Carballo, 1985; Rivas y Valle, 1987) está geográficamente encuadrada en el sector sur-oriental de la Depresión del Duero y ha sido incluida en el Vallesiense por Armenteros (1985).

- Zaratán, Las Mamblas y Villabáñez (Valladolid): (Rivas Carballo et al., 1992a; Rivas Carballo et al., 1992b; Rivas Carballo et al., 1994).

- San Cebrián de Mazote: (Valle y Civis, 1982) se encuentra en la provincia de Valladolid, en el límite con la provincia de Zamora. Se incluye en las Facies "Cuestas" y López Martínez y Sanchiz (1982) le atribuyen una edad Vallesiense basándose en los estudios de microvertebrados.

- Abezames: (Salvador de Luna, 1984; Valle y Salvador, 1985a), en la provincia de Zamora, también se incluye en las Facies "Cuestas" de edad Vallesiense (López Martínez y Sanchiz, 1982).

- Burgos: Rivas Carballo y Valle (1990) y Civis et al., (1990b) analizan varios afloramientos neógenos de la provincia de Burgos, que si bien corresponden al Mioceno, no están aún datados con exactitud. 
- Castrillo del Val: (Salvador de Luna, 1984; Valle y Salvador, 1985b), en el Vallesiense de la provincia de Burgos.

- Belorado (actualmente en estudio): en este área también se han reconocido los sistemas lacustres, pero al igual que en Torremormojón, la vegetación identificada comprende numerosos elementos subtropicales de áreas pantanosas (Taxodium, Nyssa, Clethraceae-Cyrillaceae, Bombacaceae, Sequoia...) mezclados con los elementos autóctonos. Los altos porcentajes de estos elementos megatérmicos indicarían un clima local subtropical y con un alto grado de humedad ambiental permanente, aunque no se ha determinado con exactitud si este microclima particular se debe a cercanas barreras geográficas o a un problema cronoestratigráfico, ya que aunque la posición de los afloramientos en el Mioceno no se ha podido determinar con exactitud, la mayor parte de las secciones de este área de la cuenca se incluyen en la denominada Facies "Cuestas”, que según López Martínez y Sanchiz (1982) tienen edad Vallesiense.

\section{ANDALUCÍA}

\section{Cuenca del Guadalquivir}

En esta cuenca de origen marino está representado el Mioceno Superior y el Plioceno. Las muestras correspondientes al Tortoniense están dominadas por formas herbáceas, principalmente gramíneas, y pueden asociarse a un paisaje abierto, aunque con un estrato arbóreo relativamente importante en el que destaca la familia Fagaceae. En los niveles sucesivos, el estrato arbóreo, en el que ahora se incluirían formas de clima subtropical, pasa a ser dominante junto con los helechos, lo que implica un aumento del grado de humedad y temperatura. Sin embargo, en los niveles correspondientes al Tortoniense superior se produce una brusca desaparición de las Pteridophyta y fuerte descenso de las coníferas, hechos que se asocian a un cambio de clima que pudo estar relacionado con el paso del Tortoniense al Messiniense. En el Plioceno Inferior, de nuevo hay un claro predominio del polen arbóreo y un alto porcentaje de esporas de Pteridophyta, pudiendo reflejar un medio de bosque pantanoso, en un clima cálido y húmedo (de tipo subtropical). Los afloramientos analizados se concentran en las provincias de Huelva y Sevilla, y son:

- Gibraleón: (Huelva; Peñalba, 1985; Valle y Peñalba, 1987), Tortoniense y Tránsito Tortoniense/Messiniense.

- Arroyo Trujillo: (Cantillana, Sevilla; Civis et al., 1989, 1990a; Valle y Rivas Carballo, 1990): se incluyen en el Tránsito Tortoniense/Messiniense.

- Arroyo Galapagar y Carmona: (Sevilla; Jan Du Chêne, 1977). El autor hace un estudio sintético de ambas secciones, que transcurren desde el Tortoniense superior (base de Arroyo Galapagar) hasta el Plioceno Inferior, incluyendo todos los afloramiento de Carmona en el Andaluciense (parte superior del Tortoniense superior y el Messiniense).

- Bonares: (Huelva; Peñalba, 1985; Valle y Peñalba, 1987), Zancliense.

- Casa del Pino: (Huelva; Peñalba, 1985; Valle y Peñalba, 1987), Zancliense.

\section{Arenas del Rey (Granada)}

Esta sección (Solé de Porta y Porta, 1977) fue datada como "Messiniense (=Turoliense)" (sic) por los autores basándose en la fauna de mamíferos. Palinológicamente se caracteriza por el dominio absoluto de coníferas aladas, relativa abundancia de Quercus acompañado de Nyssa, Alnus, etc. y porcentajes elevados de Pteridophyta y Gramineae, Compositae y Chenopodiaceae. Todo ello indica la presencia de una zona deprimida en el que se desarrollan las herbáceas mientras que las coníferas corresponderían a un cinturón arbóreo más o menos alejado; el clima sería de templado a cálido.

\section{Santa Inés (Málaga)}

Es un afloramiento de edad Tabianiense (Solé de Porta y Porta, 1976; Solé de Porta y Rodríguez, 1982), en el que hay un claro dominio de las formas arbóreas, especialmente Pinaceae. Las herbáceas están bien representadas, fundamentalmente por Compositae, Amaranthaceae-Chenopodiaceae y Gramineae.

\section{CATALUÑA}

\section{Vallès-Penedès (Barcelona)}

Del Mioceno del Vallès-Penedès se han analizado tres afloramientos de edad Burdigaliense superior-Langhiense: La Rierussa, S. Pau d'Ordal y Vilovi (Bessedik, 1984; Bessedik, 1985; Bessedik et Cabrera, 1985; Suc et al. 1992). Los espectros polínicos reflejan un medio abierto dominado por Compositae, Gramineae y Amaranthaceae-Chenopodiaceae y de clima cálido y seco. En general predominan las formas xéricas, especialmente Mimosaceae, y en las dos primeras localidades es destacable la existencia de un manglar con Avicennia cf. marina. Junto a estos taxa aparecen numerosos elementos megatérmicos (Bombax, Sapotaceae, Alchor$n e a, \ldots)$ que ocuparían las ripisilvas de las tierras bajas.

También en esta cuenca se han analizado los afloramientos de la Ermita de Berà (Tarragona). Se trata de unos niveles del Serravalliense que afloran próximos a la localidad de Roda de Berà (Porta et al., 1985). Los resultados palinológicos muestras una gran cantidad de microflora marina (quistes de Dinoflagelados) pero muy pocas formas continentales (principalmente Pinus y alguna Taxodiaceae).

\section{La Cerdaña (Gerona)}

En esta zona se han estudiado tres afloramientos de edad Vallesiense: Estavar, próximo a Puigcerdá (Gerona) (Jelsgerma, 1957), y Sanavastre y Sampsor (Jelsgerma, 1957; Bessedik, 1985) cercanos a la localidad de Bellver de Cerdanya (Lérida). Lo más destacable de la zona es la abundancia de Quercus, Fagus, Alnus y coníferas (Cathaya, Pinus, Taxodiaceae) que indicarían la proximidad de formaciones deciduas mixtas. En general las formas arbóreas dominan ampliamente sobre las herbáceas y han desaparecido todos los elementos megatérmicos salvo las Sapotaceae. Esta falta de registro puede ser debida al componente altitudinal ya indicado en el capítulo de macroflora. 


\section{Tarragona E2}

Se trata de un sondeo de $3.475 \mathrm{~m}$. y cuya edad va desde el Trias al Plioceno Superior (Bessais et Cravatte, 1988). El análisis palinológico se ha realizado sobre 6 niveles del Mioceno Superior (Tortoniense/Messiniense) y 21 niveles del Plioceno (Zancliense y Piacenziense). En estos no se observan grandes cambios de clima y vegetación, existiendo un notable predominio de las coníferas aladas y elementos megatérmicos.

\section{Baix Llobregat (Barcelona)}

Todos los resultados obtenidos en esta cuenca son muy similares, y los diferentes autores coinciden en que el reducido porcentaje de Taxodiaceae y la elevada proporción de formas xerófilas confirmaría la hipótesis de un ritmo climático de tipo mediterráneo, caracterizado por la desaparición progresiva de las precipitaciones estivales. Las localidades estudiadas hasta el momento son:

- Molins de Rey: (Valle, 1982).

- Papiol: (Valle y Civis, 1977; Valle, 1982; Suc, 1980; Suc et Cravatte, 1982): Zancliense.

- Plaça de les Bruixes: (Valle, 1982, 1983b).

- Can Albareda: (Valle y Civis, 1978; Valle, 1982, 1983a; Suc et Cravatte, 1982).

- San Feliu de Llobregat: (Suc, 1980; Suc et Cravatte, 1982).

\section{Ciurana (Alt Empordá, Gerona)}

Según los estudios realizados (Valle, 1982; Suc, 1980; Suc et Cravatte, 1982) podría tratarse de una paleoprovincia en la que se mantendrían los elementos megatérmicos y Taxodiaceae después de su desaparición en otras zonas de Cataluña.

\section{Garraf 1 (Barcelona)}

Un sondeo frente a las costa, del que se han analizado palinológicamente (Suc et Cravatte, 1982) tres niveles del Mioceno Superior (Tortoniense/Messiniense) y 44 del Plioceno (Zancliense y Piacenziense). En este sondeo los autores señalan un notables descenso de los árboles de climas cálidos y húmedos a medida que aumentan las formaciones herbáceas (estepas con Artemisia y Ephedra).

\section{San Onofre (Baix Ebre, Tarragona)}

En este área (Valle, 1982; Solé de Porta y Valle, 1986; Bessais et Cravatte, 1988), aunque existen taxa de carácter cálido, la escasa representación de Taxodiaceae y la abundancia de Pinus reflejan un clima estacional, con una de las estaciones más seca.

\section{PORTUGAL}

\section{Cuenca del Tajo}

Pais (1979) y Antunes and Pais (1987) hacen un resumen del Mioceno (Aragoniense y Vallesiense) de la Cuenca del Tajo, en el que incluyen además de la región de Lisboa, el Ribatejo, Cuenca del Sado y el centro-oeste de Portugal (Amor, Leiria); en el Aragoniense superior el clima es cálido y seco, con ambientes de sabana o estepa. En el Vallesiense inferior el clima se hace templado-cálido, pero sigue siendo bastante seco, al menos estacionalmente, aunque se mantendría alguna zona húmeda. La vegetación todavía está dominada por herbáceas, pero en las zonas más húmedas y en la proximidad a la costa se encuentran mayor cantidad de formas leñosas, con preponderancia de elementos templados (Populus, Salix, Quercus, Ulmus...). Ya en el Vallesiense superior los niveles de humedad siguen siendo muy bajos; aparecen extensas áreas lacustres cuyos límites variaban ampliamente de acuerdo con la estación. La vegetación refleja un ambiente estepario.

\section{Río Maior}

En los sondeos realizados en la cuenca de Rio Maior (Diniz, 1967, 1984a, 1984b) está representado todo el Plioceno. En los resultados se deja notar la influencia atlántica, con aparición de pequeñas oscilaciones climáticas (en las que están implicadas tanto temperatura como precipitaciones). Éstas marcan un cierto ritmo estacional, que difiere notablemente de otras regiones, hasta el punto que, según la autora: "al final del Plioceno permiten pensar ya en la aparición de un estilo climático cuaternario".

\section{OTROS ESTUDIOS}

\section{As Pontes (A Coruña)}

Habría que incluir aquí los resultados obtenidos por Medus et Nonn (1963) y Medus (1965a, 1965b) en Galicia, puesto que el autor los incluye en el Mioceno Superior. Sin embargo, no solo la edad que propone, Tortoniense-Pontiense, es imprecisa, sino que los datos que proporciona el análisis palinológico, especialmente los porcentajes de Simarubaceae (que según Bessedik, 1984, desaparecen del Mediterráneo nor-occidental en el tránsito BurdigalienseLanghiense) hacen pensar que la edad de los afloramientos es mucho anterior. Esto coincide con los datos microfaunísticos, que datan la Cuenca de As Pontes como Oligoceno superior (López Martinez et al., 1993).

\section{Venta del Moro (Valencia)}

El estudio de este afloramiento se debe a Van Campo (1976, 1989). Según la autora, la característica más importante de la vegetación es la persistencia de los taxa de herbáceas que se han mantenido hasta la actualidad, mientras que entre las formas leñosas destacan la abundancia de Abietaceae y la presencia de elementos tropicales. El conjunto polínico, y en especial el gran número de herbáceas sugieren un paisaje relativamente abierto con una ripisilva lujuriante y un bosque de coníferas a mayor altitud. Van Campo (op. cit.) sugiere una edad Vallesiense superior para la microflora, aunque Mathisen and Morales (1981) datan el afloramiento como Turoliense superior basándose en la fauna de mamíferos. 


\section{BIBLIOGRAFIA}

Almera, J. 1891. Descubrimiento de tres floras terciarias en nuestros alrededores. Crónica científica, 14, 476-479.

Almera, J. 1894. Descripción de los depósitos pliocénicos de la cuenca del Bajo Llobregat y llano de Barcelona. Memorias de la Real Academia de Ciencias y Artes de Barcelona, 3, 321-351.

Almera, J. 1899. Compte rendu de l'excursion du 28 de septiembre a Sans et a Montjuich. Bulletin de la Societé Geologique de France, 26, 680-689.

Álvarez Ramis, C. 1975. Quelques considérations ecologiques sur le gisement vallesien de Can Llobateres (Barcelone, Espagne). Comptes Rendus du 100e Congrès National des Societes Savantes, Paris, II, 11-16.

Álvarez Ramis, C. 1977. Nuevas especies de fanerógamas del Plioceno de Papiol. Estudios Geológicos, 33, 119-121.

Álvarez Ramis, C. 1981. Paleoclima de las cuencas pontienses del Pirineo Catalán. Anais II Congreso Latino-Americano de Paleontologia, Porto Alegre, 553-564.

Álvarez Ramis, C. et Doubinguer J. 1981. Sur la paleobotanique du gisement miocène de Buñol (Valencia, Espagne). Boletín de la Real Sociedad Española de Historia Natural. Sección Geológica, 79, 173- 179.

Álvarez Ramis, C. y Fernández Marrón, M. T. 1975. Estudio paleobotánico y datación estratigráfica del yacimiento terciario de Frías (Burgos). Primer Centenario de la Real Sociedad Española de Historia Natural. Sección Geológica, 1, 27-41.

Álvarez Ramis, C. et Fernández Marrón, M. T. 1984. Les points de vue qualitatif et quantitatif en paleophytologie. Application à la connaissance du paleoclimat du gisement pliocene de Papiol, Espagne. Paléobiologie continentale, 14, 69-73.

Álvarez Ramis, C. y Fernández Marrón, M. T. 1994. Conexiones establecidas entre los palinomorfos y los macrorrestos vegetales del Mioceno Medio de Rubielos de Mora (Teruel). In: Polen y esporas: contribución a su conocimiento. (Ed. I. La-Serna Ramos), Universidad de La Laguna, Tenerife, 323-331.

Álvarez Ramis, C. y Golpe-Posse, J. M. 1981. Sobre la paleobiología de la cuenca de Cerdanya (depresiones pirenaicas). Boletín de la Real Sociedad Española de Historia Natural. Sección Geológica, 79, 31-44.

Antunes, M. T. and Pais, J. 1987: Climate during Miocene in Portugal and its evolution. Paléobiologie Continentale, 14, 75-89.

Areitio y Larrinaga, A. 1874. Enumeración de plantas fósiles españolas. Anales de la Sociedad Española de Historia Natural, 3 , 225-259.

Armenteros, I. 1985: Estratigrafía y sedimentología del Neógeno del sector suroccidental de la Depresión del Duero (Aranda de Duero-Peñafiel). Tesis Doctoral, Universidad de Salamanca, $692 \mathrm{p}$.

Barrón, E. 1992a. Presencia de Fraxinus excelsior Linne (Oleaceae, Gentianales) en el Mioceno Superior de la Depresión Ceretana. Implicaciones tafonómicas y paleoecológicas. Revista Española de Paleontología, 7, 101-108.

Barrón, E. 1992b. La presencia del genero Acer, L. (Aceraceae, Sapindales, Rosidae) en el Mioceno Superior de la Depresión Ceretana (Lérida). In: Historia Natural '91. (Ed. A. Alemany). Universitat de les Illes Balears, Palma de Mallorca, 543-551.

Barrón, E. and Diéguez, C. 1994. Neogene species of the genus Fagus L. from La Cerdaña (Lérida, Spain). Taxonomic conclusions and phylogenetic considerations. Anales del Jardín Botánico de Madrid, 52, 21-32.
Bessais, E. et Cravatte, J. 1988: Les écosystèmes végétaux pliocènes de Catalogne méridionale. Variations latitudineles dans le domaine nord-ouest méditerranéen. Geobios, 21, 49-63.

Bessedik, M. 1984. The early Aquitanian and upper Langhian-lower Serravallian environments in the northwestern mediterranean region. Paléobiologie Continentale, 14, 153-179.

Bessedik, M. 1985. Reconstitution des environnements miocènes des regions nord-ouest mediterranéennes à partir de la palynologie. Thèse d'État. Université des Sciences et Techniques du Languedoc, 162 p.

Bessedik, M. et Cabrera, L. 1985. Le couple récif-mangrove à Sant Pau d'Ordal (Vallès-Penedès, Espagne), tèmoin du maximum transgressif en Mediterranée nord-occidentale (Burdigalien supérieur-Langhien inférieur). Newsletter of Stratigraphy, 14, 20-35.

Calvo, J. P, Daams, R., Morales, J., López-Matínez, N., Agustí, J., Anadón, P., Armenteros, I., Cabrera, L., Civis, J., Corrochano, A., Díaz-Molina, M., Elizaga, E., Hoyos, M., Martín-Suárez, E., Martínez, J., Moissenet, E., Muñoz, A., Pérez-García, A., Pérez-González, A., Portero, J. M., Robles, F., Santisteban, C., Torres, T., Van der Meulen, A. J., Vera, J. A. and Mein, P. 1993. Up-to-date Spanish continental Neogene synthesis and paleoclimatic interpretation. Revista de la Sociedad Geológica de España, 6, 29-40.

Civis, J., Flores, J. A., Francés, G., González Delgado, J. A., Sierro, F. J. et Valle, M. F. 1989. Études paleontologiques dans le Neogène du Bassin du Guadalquivir (Espagne) et dans des forages adjacents, leur relation dans les domaines atlantique et mediterrannéen. $1^{o}$ Colloque du Neogene Atlantico-Mediterraneen, Guide excursions et Résumé des Communications, 33-38.

Civis, J., González-Delgado, J. A., Andrés, I., Valle, M. F., Sierro, F. J., Flores, J. A. y Dabrio, C. J. 1990a. El contenido paleontológico de la sección Tortoniense-Messiniense de Arroyo Trujillo. In: Actas de Paleontología (Eds. J. Civis Llovera y J.A. Flores Villarejo), Universidad de Salamanca, 85-101.

Civis, J., Sierro, F. J., Flores, J.A., Andrés, I., Gonzales Delgado, J. A., Valle, M.F. y Rivas Carballo, M.R. 1990b. Estudio de los Invertebrados y Palinomorfos del Paleógeno y Neógeno en la provincia de Burgos. Obra Social de la Caja de Ahorros Municipal de Burgos (Inédita). 127 p.

De Bruijn, H., Daams, R., Daxner-Höck, G., Fahlbusch, V., Ginsburg, L., Mein, P. and Morales, J. 1992. Report of the RCMNS working group on fossil mammals, Reisensburg 1990. Newsletters in Stratigraphy, 26, 65-118.

Diniz, F. 1965. Note preliminaire sur la flore pollinique du gisement d'Espadonal à Rio Maior (Portugal). Pollen et Spores, 7, 373-779.

Diniz, F. 1967: Una flore tertiaire du caracter méditerranéen au Portugal. Review of Palaeobotany and Palynology, 5, 263-268.

Diniz, F. 1984a: Apports de la palynologie à la connaissance du Pliocene Portugais. Rio Maior: un bassin de reference pour l'histoire de la flore, de la vegetation et du climat de la façade atlantique de l'Europe meridionale. These d'etat, Université Sciences et Techniques du Languedoc, 230 p.

Diniz, F. 1984b. Étude palynologique du bassin pliocène de Rio Maior (Portugal). Paléobiologie Continentale, 14, 259-267.

Fernández Marrón, M. T. 1970. Aportación al estudio paleobotánico y paleoecológico de la flora terciara de Olocau (Valencia) Boletín de la Real Sociedad Española de Historia Natural. Sección Geológica, 68, 131-144. 
Fernández Marrón, M. T. 1971. Estudio paleoecológico y revisión sistemática de la flora fósil del Oligoceno español. Publicaciones de la Facultad de Ciencias. Universidad Complutense de Madrid, 177 p.

Fernández Marrón, M. T. 1972. Nota sobre la paleoecología del yacimiento de Libros (Teruel) en relación con los Artropodos y las plantas fósiles del biotopo. Acta Geológica Hispánica, VII, 149-153.

Fernández Marrón, M. T. 1979. Essai de résolution de problemes stratigraphiques de la limite Paleogene-Neogene par les études de macroflore. Annales Geológiques des Pays Helleniques, tome hors de sèrie, I, 403-412.

Fernández Marrón, M. T. y Álvarez Ramis, C. 1967. Contribución al estudio de las Gimnospermas fósiles del Oligoceno de Ribesalbes (Castellón). Estudios Geológicos, XIII, 155-161.

Ferrer, J., Fernández Marrón, M. T. y Liñán, E. 1993. Nota sobre las plantas fósiles del Mioceno de Épila (Zaragoza). Geogaceta, 14, 94-97.

García Moreno, E. 1983. Paleontología y estratigrafía de la zona central de la Cuenca del Duero. Estudio de los Micromamíferos del Mioceno medio de la serie de Torremormojón (Palencia). Tesis de Licenciatura, U.C.M., 200 p. (Inédita).

Heer, O. 1881. Contributions à la flore fossile du Portugal. Sections des travaux Geologiques du Portugal. Academie Royale de Sciences. Lisbonne, 51 p.

Jan du Chêne, R. 1977. Étude palynologique du Miocène supérieur andalou (Espagne). Revista Española de Micropaleontología, 9, 97-114.

Jelsgerma, S. 1957. Investigaciones palinológicas de lignitos terciarios procedentes de Cerdaña y del Valle de Arán (Pirineos españoles). Cursillos y Conferencias del Instituto Lucas Mallada, 4, 159-162.

López Martínez, N. y Sanchiz, B. 1982: Los primeros microvertebrados de la Cuenca del Duero: listas faunísticas preliminares e implicaciones bioestratigráficas y paleofisiográficas. $I^{a}$ Reunión sobre Geología de la Cuenca del Duero, Salamanca, 1979, I.G.M.E., 1. ${ }^{a}$ parte: $341-353$.

López Martínez, N., García Moreno, E. y Álvarez Sierra, M. A. 1986: Palentología y bioestratigrafía (Micromamíferos) del Mioceno medio y superior del sector central de la Cuenca del Duero. Stvdia Geologica Salmanticensia, 22, 191-212.

López-Martinez, N., Fernández Marrón, M. T., Pelaez-Campomanes, P. y De La Peña Zarzuelo, A. 1993. Estudio paleontológico en las cuencas terciarias de Galicia. Revista de la Sociedad Geológica de España, 6, 19-28.

Mathisen, M. and Morales, J. 1981. Stratigraphy, facies and depositional environments of the Venta del Moro vertebrate locality, Valencia, Spain. Estudios Geológicos, 37, 199-207.

Medus, J. 1965a. Contribution palynologique à la connaissance de la flore et de la végétation neogène de l'ouest de l'Espagne. Thèse $3 \mathrm{e}$ cycle, Montpellier, $91 \mathrm{p}$.

Medus, J. 1965b. L'evolution biostratigraphique d'une lagune neogène de Galicie (Espagne). Pollen et Spores, 7, 381-393.

Medus, J. et Nonn, H. 1963: Premières results d'analyses polliniques à Puentes de García Rodriguez (Province de Galice, Espagne) et conclussions geomorphologiques qui en d'écouter. Comptes Rendus de l' Academie des Sciences, 256, 1500-1572.

Meléndez Meléndez, B. 1945. Contribución al estudio de la flora fósil del Mioceno de Libros (Teruel). Anales del Jardín Botánico de Madrid, 6, 105-107.
Menéndez Amor, J. 1951. Una piña fósil nueva para el Plioceno de Málaga. Boletín de la Real Sociedad Española de Historia Natural. Sección Geológica, 49, 193-195.

Menéndez Amor, J. 1955. La depresión Ceretana española y sus vegetales fósiles. Característica fitopaleontológica del Neógeno de la Cerdaña española. Memorias de la Real Academia de Ciencias exactas, físicas y naturales de Madrid, serie Ciencias Naturales, 18, 1-344.

Pais, J. 1972. Fósseis de Quercus suber L. provenientes de formações cenozóicas continentais do Alentejo. Boletim de Museo e Laboratorio mineralogico de la Facultade de Ciencias, 13, 35-41.

Pais, J. 1973a. Sur un bois d'annonaceae tertiaire du Portugal: Annonoxylon texeirae n. sp. Boletim da Sociedade Geologica de Portugal, 18, 171-178.

Pais, J. 1973b. Vegetais fosseis de Ponte de Sor. Boletim da Sociedade Geologica de Portugal, 18, 123-135.

Pais, J. 1979. La végétation de la basse vallee du Tage (Portugal) au Miocène. Annales Geologiques des Pays Helleniques, tome hors de serie, II, 933-942.

Pais, J. 1983. Vegetais de Vale de Coelheiros (Grândola-formaçao de Marateca). Ciências da Terra (U.N.L.), 7, 129-140.

Peñalba, C. 1985: Estudio esporopolínico del Neógeno occidental de la Cuenca del Guadalquivir. Tesis de Licenciatura, Universidad de Salamanca, $168 \mathrm{p}$.

Porta, J. de, Civis, J., Sierro, F. J., Solé, N. y Flores, J. A. 1985. Áspectos bio y Cronoestratigráficos del Mioceno de Berà (Tarragona). Estudios Geológicos, 41, 413-417.

Rérolle, M. L. 1884-1885. Études sur les vegetaux fossiles de Cerdagne. Revue des Sciences Naturelles de Montpellier, (3)IV, 167-191, 252-298, 368-386.

Rivas Carballo, M. R. 1985: Palinología del Neógeno en la Cuenca del Duero (Torremormojón, Palencia, y Peñafiel, Valladolid). Tesis de Licenciatura. Universidad de Salamanca, 111 p.

Rivas Carballo, M. R. 1987: Análisis palinológico del tramo superior de la sección de Torremormojón (Palencia). In: Actas de Palinología (eds. J. Civis y M.F.), Universidad de Salamanca, 337-344.

Rivas Carballo, M. R. 1989. Palinología del Neógeno de la región sur-oriental de la Depresión del Duero (Aranda de DueroPeñafiel). Tesis Doctoral, Universidad de Salamanca, 244 p.

Rivas Carballo, M. R. 1991a. La vegetación y el clima en el Mioceno (Aragoniense superior-Vallesiense) en la región sur-oriental de la Depresión del Duero (España). Boletín de la Real Sociedad Española de Historia Natural, Sección Geológica, 86, 53-64.

Rivas Carballo, M. R. 1991b. The development of vegetation and climate during the Miocene in the Southeastern sector of the Duero Basin (Spain). Review of Palaeobotany and Palynology, 67, 341-351.

Rivas Carballo, M. R. y Valle, M. F. 1986: Nuevas aportaciones a la Palinología del Terciario de la Cuenca del Duero. Torremormojón (Palencia). Stvdia Geologica Salmanticensia, 22: 133-143.

Rivas Carballo, M. R. y Valle, M. F. 1987: Palinología del Neógeno de la Cuenca del Duero. Peñafiel (Valladolid). In: Actas de Palinología (eds. J. Civis y M.F. Valle), Universidad de Salamanca, 345-350.

Rivas Carballo, M. R. y Valle, M. F. 1990. Estudio del polen, esporas y palinomorfos del Paleógeno y Neógeno de la provincia de Burgos. In: Polen, Esporas y sus aplicaciones (eds. G. Blanca et. al.), 445-451. 
Rivas Carballo, M. R., Alonso Gavilán, G., Civis, J., González Delgado, J. A. y Valle, M. F. 1992a. Palinoflora e Invertebrados (Moluscos y Ostrácodos) de los últimos 12 millones de años en los alrededores de Valladolid. Universidad de Salamanca, 52 p. (Inédita).

Rivas Carballo, M.R., Alonso Gavilán, G., Civis, J. y Valle, M.F. 1992b. Paleoclimatología y paleoecología de las facies de Zaratán, Neógeno de la Cuenca del Duero (provincia de Valladolid, España). In: Actas del III Congreso Geológico de Espa$\tilde{n} a$ (eds. J. Uris, F.J. Sierro, y L.C. Sánchez de Posada), Salamanca, 1, 539-543.

Rivas Carballo, M. R., Alonso Gavilán, G., Valle, M. F. and Civis, J. 1994. Miocene palynology of the central sector of the Duero Basin in relation to palaeogeography and palaeoenvironment. Review of Palaeobotany and Palynology, 82, 251-264.

Roiron, P. 1983. Nouvelle étude de la macroflore Plio-Pleistocène de Crespià (Catalogne, Espagne). Geobios, 16, 687-715.

Salvador de Luna, J.V. 1984: Análisis esporopolínico en el borde suroccidental y nororiental del Neógeno de la Cuenca del Duero (Abezames -Zamora- y Castrillo del Val -Burgos). Tesis de Licenciatura, Universidad de Salamanca, 168 p. (Inédita).

Sanz de Siria Catalán, A. 1979. Flora miocenica de Espiells, Cuenca del Penedès (Nota preliminar). Butlletí Informatiu. Institut de Paleontología de Sabadell, any XI, 48-54.

Sanz de Siria Catalán, A. 1980a. Plantas miocénicas de Sanavastre (Gerona). Depresión de Cerdaña. Butlletí Informatiu. Institut de Paleontología de Sabadell, any XII, 45-50.

Sanz de Siria Catalán, A. 1980b. Estudio sistemático y paleoecológico de la flora miocénica de la Cuenca de La Seo d'Urgel. Paleontología i Evolució, 14, 1-28.

Sanz de Siria Catalán, A. 1981a. Flora del Mioceno superior de La Bisbal (Baix Empordá). Butlletí Informatiu. Institut de Paleontología de Sabadell, any XIII, 57-68.

Sanz de Siria Catalán, A. 1981b. La flora burdigaliense de los alrededores de Martorell (Barcelona). Paleontología i Evolució, 16, 3-13.

Sanz de Siria Catalán, A. 1982a. La flora del Mioceno medio de las cercanias de Martorell. Butlletí Informatiu. Institut de Paleontología de Sabadell, any XIV, 49-61.

Sanz de Siria Catalán, A. 1982b. La flora pliocénica de Siurana (Gerona). Paleontología i Evolució, 17, 3-14.

Sanz de Siria Catalán, A. 1983-1984a. Aportaciones al conocimiento de la flora pliocénica de los alrededores de Papiol (Barcelona). Paleontología i Evolució, 18, 151-160.

Sanz de Siria Catalán, A. 1983-1984b. La flora miocénica de los alrededores de Sant Sadurní d'Anoia (Barcelona). Paleontología i Evolució, 18, 161-172.

Sanz de Siria Catalán, A. 1985. Datos para el conocimiento de las floras pliocénicas de Cataluña. Paleontología i Evolució, 19, 167-177.

Sanz de Siria Catalán, A. 1987. Datos para el conocimiento de las floras pliocénicas de Cataluña. Paleontología i Evolució, 21, 295-303.

Sanz de Siria Catalán, A. 1988. Los vegetales miocénicos de Rubí (Barcelona). Paleontología i Evolució, 22, 71-76.

Solé de Porta, N. y Porta, J. de. 1976. Datos palinológicos y faunísticos del Plioceno inferior de Santa Inés (Málaga, España). Cuadernos de Geología, 7, 53-55.
Solé de Porta, N. y de Porta, J. 1977: Primeros datos palinológicos del Messiniense (=Turoliense) de Arenas del Rey (Granada). Stvdia Geologica Salmanticensia, 13: 67-88.

Solé de porta, N. y Rodríguez, P. 1982. Características palinológicas y Foraminíferos planctónicos del Tabianiense de Santa Inés (Málaga, España). Stvdia Geologica Salmanticensia, 17, 105-115.

Solé de Porta, N. y Valle, M. F. 1986. Estudio palinológico del Plioceno de San Onofre (Tarragona). Estudios Geológicos, 42, 63-66.

Suc, J. P. 1980. Contribution à la connaissance du Pliocène et du Pleistocène Infèrieur des régions mediterranéennes d'Europe Occidentale par l'analyse palynologique des depots du Languedoc-Roussillon (Sud de la France) et de la catalogne (Nord-Est de l'Espagne). Thèse d'État. Université des Sciences et Techniques du Languedoc, 198 p.

Suc, J. P. et Cravatte, J. 1982: Etude palynologique du Pliocène de Catalogne (Nord-est de l'Espagne). Apports à la connaissance de l'histoire climatique de le Mediterranée occidentale et implications chronostratigraphiques. Paléobiologie Continentale, 13, 1-31.

Suc, J. P., Clauzon, G., Bessedik, M., Leroy, S., Zheng, Z., Drivaliari, A., Roiron, P., Ambert, P., Martinell, J., Domenech, R., Matías, I., Julià, R. and Anglada, R. 1992. Neogene and Lower Pleistocene in Southern France and Northeastern Spain, Mediterranean environments and climate. Cahiers de Micropaléontologie, 7, 165-186.

Teixeira, C. 1946. Revisão da flora fossil de Azambuja. Boletim de Mineralogia e Geologia da Universidade de Lisboa, 14, 3-15.

Teixeira, C. 1947a. A flora pliocenica de Montijo. Comunicaçoes dos Serviços Geologicos de Portugal, 28, 213-216.

Teixeira, C. 1947b. Flora de Senhora da Saude, Santarém. Comunicaçoes dos Serviços Geologicos de Portugal, 28, 145-147.

Teixeira, C. 1947c. Note sur une pomme de pin du Miocène de Lisbone. Comunicaçoes dos Serviços Geologicos de Portugal, 28, 129-132.

Teixeira, C. 1952a. Flora fossil das argilas de Vale do Carros (Alpiarça). Comunicaçoes dos Serviços Geologicos de Portugal, 33, 11-14.

Teixeira, C. 1952b. Flora fossil do Miocenico de Esbarrondadoiro, Odivelas. Comunicaçoes dos Serviços Geologicos de Portugal, 33, 93-97.

Teixeira, C. 1952c. Flora fossil do Pliocenico de Vale de Santarém. Comunicaçoes dos Serviços Geologicos de Portugal, 33, 51-64.

Teixeira, C. 1973a. Oreodaphne heeri Gaud., une nouvelle espèce fossile du Pliocène de Rio Maior. Boletim da Sociedade Geologica de Portugal, 18, 147-149.

Teixeira, C. 1973b. Quelques cònes de pin fossile du Miocène portugais. Boletim da Sociedade Geologica de Portugal, 18, 151-154.

Valle, M. F. 1982. Estudio palinológico del Plioceno del NE de España. Tesis Doctoral, Universidad de Salamanca, 204 p.

Valle, M. F. 1983a. Nuevas aportaciones palinológicas al Plioceno de Can Albareda (Barcelona). Stvdia Geologica Salmanticensia, 19: 151-159.

Valle, M. F. 1983b. Palinología del Plioceno de la Placa de les Bruixes (Barcelona). Stvdia Geologica Salmanticensia, 19, 133-137.

Valle, M. F. y Civis, J. 1977. Análisis palinológico preliminar en el Plioceno de Papiol (Barcelona). Stvdia Geologica Salmanticensia, 13, 56-66. 
Valle, M. F. y Civis, J. 1978. Investigaciones palinológicas en el Plioceno inferior de Can Albareda (Barcelona). Palinología, $n^{\circ}$ est. 1, 463-468.

Valle, M. F. y Civis, J. 1982. Palinología de las facies "Cuestas" en el borde occidental de la Cuenca del Duero. In: Actas del IV Simposio A.P.L.E., (eds. N. Solé y M. Suárez Cervera), Barcelona, 351-361.

Valle, M. F. y Peñalba, C. 1987: Aspectos palinológicos en el Neógeno del Suroeste de España. In: Paleontología del neógeno de Huelva (W. Cuenca del Guadalquivir). (Ed. Dpto. de Paleontología), Universidad de Salamanca, 153-158.

Valle M. F. y Rivas Carballo, M. R. 1990. Palinología del Tránsito Tortoniense-Messiniense en las facies neógenas del borde norte de la Cuenca del Guadalquivir (E. de Sevilla). In: Polen, Esporas y sus aplicaciones (eds. G. Blanca et. al.), 453-457.

Valle, M. F. y Salvador, J. V. 1985a. Resultados palinológicos en el borde sur-occidental de la Cuenca del Duero. Abezames (Zamora). Estudios Geológicos, 41, 69-75.
Valle, M. F. y Salvador, J. V. 1985b. Palinología del Neógeno de la Cuenca del Duero. Castrillo del Val (Burgos). Estudios Geológicos, 41, 237-241.

Van Campo, E. 1976. La flore sporopollinique du gisement miocène terminal de Venta del Moro (Espagne). Thèse de Licenciature. Universitat du Languedoc, $54 \mathrm{p}$.

Van Campo, E. 1989. La flore pollinique du Miocène terminal de Venta del Moro (Espagne). Acta palynologica, 1, 9-32.

Villalta, J. F. y Crusafont, M. 1945. La flora miocénica de la Depresión de Bellver. Ilerda, 3, 339-353.

Villalta, J. F. y Vicente, J. 1972. Una flora del Cuaternario antiguo en las cercanias de Crespià. Acta Geológica Hispánica, 7, 120-128.

Vicente i Castells, J. 1988. La flora fósil de Montjuïc (Barcelona). Societat d'Historia Natural, Sta. Coloma de Gramenet. Sèrie Monográfica, 1, 1-93.

Vigón, M. A. 1980. Nuevas aportaciones al estudio paleoecológico del yacimiento mioceno de Ribesalbes. Tesis de Licenciatura, Universidad Complutense de Madrid, 67 p. (Inédita).

Manuscrito recibido: 10 de enero, 1995 Manuscrito aceptado: 22 de junio, 1995 